\title{
Towards an understanding of hole superconductivity
}

\author{
J. E. Hirsch \\ Department of Physics, University of California, San Diego, La Jolla, CA 92093-0319
}

\begin{abstract}
From the very beginning K. Alex Müller emphasized that the materials he and George Bednorz discovered in 1986 were hole superconductors. Here I would like to share with him and others what I believe to be the key reason for why high $T_{c}$ cuprates as well as all other superconductors are hole superconductors, which I only came to understand a few months ago. This paper is dedicated to Alex Müller on the occasion of his 90th birthday.
\end{abstract}

PACS numbers:

\section{INTRODUCTION}

The very first paper by K. Alex Müller listed in Web of Science, from when he was a youthful 27-year-old, is on an apparatus to measure Hall effect [1]. It shows that from the very beginning of his scientific career, Alex Müller was well aware of the difference between electrons and holes. [2]

The concept of holes has played a prominent role in semiconductor physics for a long time, as exemplified by the title of Shockley's 1950 book "Electrons and holes in semiconductors". It also of course has played a prominent role in quantum electrodynamics since Dirac dis-

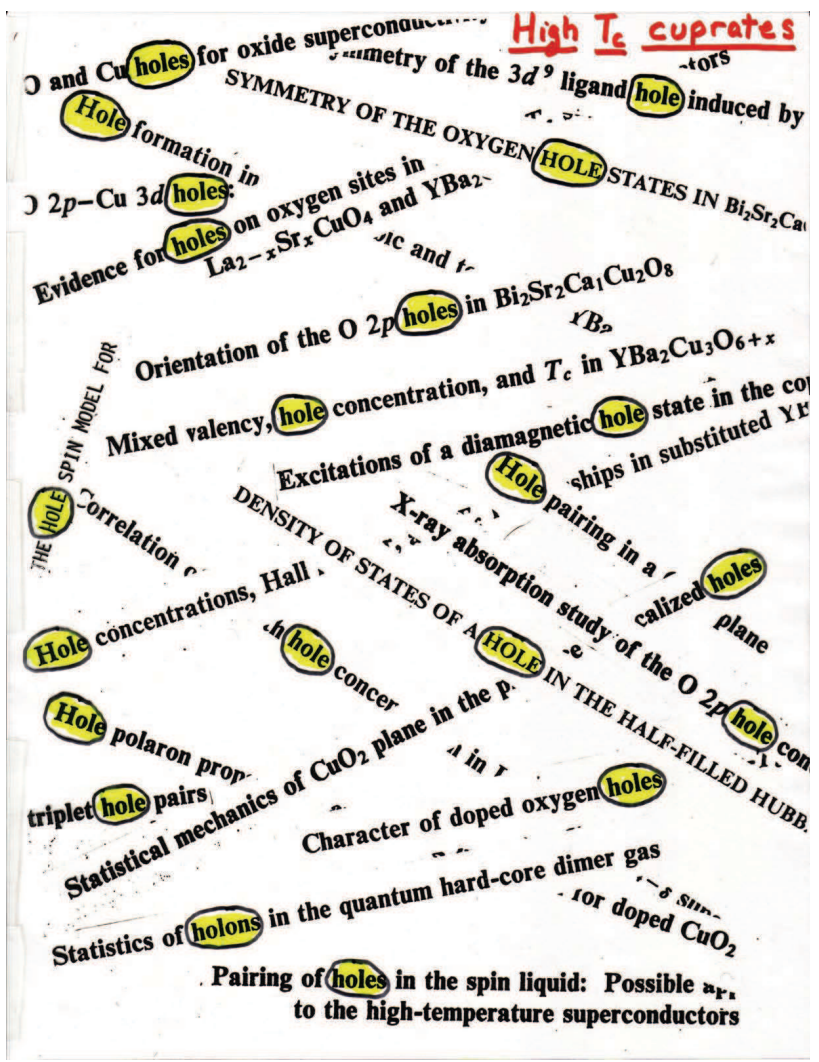

FIG. 1: Titles of some papers published in the early days of high $T_{c}$ research (see text). covered holes in 1930. But it had played essentially no role in superconductivity until the discovery of high $T_{c}$ cuprates.

In his 1987 paper in LT18, 'A Road towards High $T_{c}$ Superconductivity' [3] Alex spelled it out clearly for the first time: "Basically, all these materials, are hole superconductors." Again in his 1987 Science paper 'The Discovery of a Class of High-Temperature Superconductors' [4] Alex remarks "This new class of materials found at the IBM Zurich Research Laboratory are hole rather than electron superconductors". In his 1988 paper in the proceedings of the NEC Symposium on Mechanisms of High Temperature Superconductivity he states [5] "As the $T_{c}$ of hole-containing $\mathrm{BaBiO}_{3}$ is more than twice as high as that of the electron-containing compound, one might expect an enhancement of $T_{c}$ for hole superconductivity over electron superconductivity in the cuprates if the latter are found." It is clear that Alex was struck by the realization that holes seemed to be favorable for superconductivity, which was not part of his initial theoretical views on high temperature superconductivity (JahnTeller polarons) that guided him and Bednorz in their search and led them to their discovery. Thus, he emphasized the 'hole' aspect in many of his early papers and talks. The era of hole superconductivity had begun.

Soon thereafter, everybody working on cuprates was talking about 'holes'. Figure 1 shows a transparency I made in those early days with random titles of papers that I used in talks to emphasize this point.

I met Alex Müller for the first time at the NEC symposium in late 1988 and listened keenly to his talk, one of the first in the program. I still have the handwritten notes I took at the time, 6 pages of them. In the middle of the second page there is the statement "These materials are hole superconductors", with "hole superconductors" underlined. I still remember vividly the emphasis he put on those words in his presentation, that deeply impressed me at the time.

Later in that meeting, $H$. Takagi made a comprehensive presentation of transport properties of $\left(\mathrm{La}_{1-x} \mathrm{Sr}\right)_{2} \mathrm{CuO}_{4}$ [6]. He showed a slide of $T_{c}$ versus hole concentration $(p)$ and asked "why does superconductivity disappear" at $p=0.15 ?$ ? On the very next slide he showed a graph of Hall coefficient versus hole concentration showing that it changes sign from positive 
to negative precisely at $p=0.15$.

Takagi suggested in this presentation that $T_{c}$ goes to zero in the overdoped regime because of a cross-over from Mott-Hubbard to Fermi liquid regime. He did not, to the best of my recollection and according to my notes taken at the time, directly connect the change in sign of the Hall coefficient from positive to negative to the disappearance of superconductivity. I wondered for a long time why he hadn't done that and only much later I learned why [7]. It turns out that at that time, October 1988, he and his coworkers already had discovered the so-called 'electron superconductors'. He did not mention this discovery at that meeting nor did other speakers, those results were announced in January 1989 [8]. But this clearly must have been the reason why he did not think that the type of charge carrier (whether hole or electron) was a determining factor. It took many more years and a lot of experiments to establish that the electron-doped cuprates are in fact also hole superconductors [9].

In the paragraph above I said the concept of holes had played essentially no role in superconductivity. The caveat is because in fact several researchers in the early days of superconductivity did suggest that a positive Hall coefficient was favorable to superconductivity [10]. However, the concept fell completely out of favor after the establishment of the BCS theory of superconductivity, within which the character of the carriers, whether electrons or holes, plays no role.

Ever since I heard that fateful talk by Alex Müller in 1988 I have been convinced that hole carriers are essential for superconductivity in all materials [11], not just in high $T_{c}$ cuprates. Together with Frank Marsiglio and other coworkers we have presented many arguments and calculations in favor of this hypothesis [12]. In this short paper I would like to discuss what I think is the most fundamental reason why holes are indispensable for superconductivity, that I have only understood a few months ago. But first some preliminaries.

\section{HOLES IN CONDENSED MATTER PHYSICS}

The concept of holes in solids was introduced by Werner Heisenberg in 1931 [13]. He writes: "Die Elektrizitlätsleitung in Metallen mit einer geringen Anzahl von Löchern kann also in jeder Beziehung beschrieben werden wie die Leitung in Metallen mit einer geringen Anzahl von positiven Leitungselektronen. Daraus folgt unmittelbar der anomale Halleffekt fur solche Metalle." Similarly Peierls in 1932 writes [14] "Ein Band, in dem sich nur wenige Elektronen befinden, verhalten sich in jeder Beziehung genau so, wie ein Band, in dem nur für wenige Elektronen noch Platz ist, mit dem Unterschied, dass den freien Plätzen eine umgekehrte - also positive - Ladung zuzuschreiben ist. Da jedoch die Leitfähigkeit unabhängig vom Vorzeichen der Ladung ist, wird sich dieser Unterschied zunächst in der Leitfähigkeit noch nicht bernerkbar machen." The reason for the 'anomalous' (positive) Hall coefficient in metals with 'hole' carriers was worked out by Peierls upon the suggestion of Heisenberg already in 1929 [15].

Both of the above statements say that "in jeder Beziehung", i.e. "in every respect", holes are just like electrons in solids. This point of view has been pervasive in condensed matter physics ever since. Yet it is incorrect. If it was correct, there would be no superconductivity.

In a paper I wrote in 2005 I listed many reasons why holes are not like electrons, as shown in Figure 2. The most relevant one regarding superconductivity is highlighted. I will explain this in a later section.

TABLE I. Different properties of the carriers at the Fermi energy when the Fermi level is near the bottom (bonding electron) and near the top of the band (antibonding electron).

\begin{tabular}{|c|c|}
\hline $\begin{array}{l}\text { Bonding electron } \\
\text { at the Fermi energy }\end{array}$ & $\begin{array}{l}\text { Antibonding electron } \\
\text { at the Fermi energy }\end{array}$ \\
\hline Undressed & Dressed \\
\hline Low kinetic energy & High kinetic energy \\
\hline Long wavelength & Short wavelength \\
\hline Small effective mass & Large effective mass \\
\hline Uniform charge density & Nonuniform charge density \\
\hline Moves in direction of force & Moves opposite to force \\
\hline Conducts electricity & Anticonducts electricity \\
\hline Contributes to Drude weight & Anticontributes to Drude weight \\
\hline Detached from lattice & Transfers momentum to lattice \\
\hline Large quasiparticle weight & Small quasiparticle weight \\
\hline Coherent conduction & Incoherent conduction \\
\hline Large Drude weight & Small Drude weight \\
\hline Negative Hall coefficient & Positive Hall coefficient \\
\hline Good metals & Bad metals \\
\hline Stable lattices & Unstable lattices \\
\hline Ions attract each other & Ions repel each other \\
\hline Carriers repel each other & Carriers attract each other \\
\hline Normal metals & Superconductors \\
\hline
\end{tabular}

FIG. 2: From a paper the author wrote in 2005 [16]. The most important reason for why holes are necessary for superconductivity is highlighted.

\section{ELECTRON-HOLE ASYMMETRIC POLARONS AND DYNAMIC HUBBARD MODELS}

Alex Müller has always focused on polarons as being at the root of high temperature superconductivity [1719]. Somewhat overlapping with his idea, within the theory of hole superconductivity, high $T_{c}$ originates in small electron-hole asymmetric polarons [20, 21]. In our view the dominant polaronic aspect arises from electronelectron interactions rather than from electron-lattice interactions. Nevertheless, even for electron-phonon po- 
larons electron-hole asymmetry can play a big role and favor hole over electron superconductivity [22], consistent with Alex's expectations.

A very simple, natural and general extension of the conventional Hubbard model leads to 'dynamic Hubbard models' and electron-hole asymmetric electronic polarons. In the conventional Hubbard model, electrons in doubly occupied orbitals pay the Coulomb repulsion price $U$, but their orbitals are unmodified relative to the singlyoccupied orbital. However, in reality a doubly occupied atomic orbital expands relative to the singly-occupied orbital, due to electron-electron repulsion. This 'orbital relaxation' causes a reduction of the bare $U$ and leads to electron-hole asymmetry. Dynamic Hubbard models describe this physics [23]. When a hole propagates, the orbital relaxation causes 'dressing' of the quasiparticle and effective mass enhancement, as in small polarons. Instead, when an electron propagates no such effects exist. The effects are largest when the effective ionic charge is small, so that the modification of the orbital upon double occupancy is large. Figure 3 shows a 'cartoon picture' of the physics that is included in dynamic Hubbard models and not in the conventional Hubbard model. The orbital expansion lowers the electronic kinetic energy (as well as the Coulomb repulsion), and causes negative charge to expand outward. Both aspects are relevant to the physics of hole superconductivity [24].

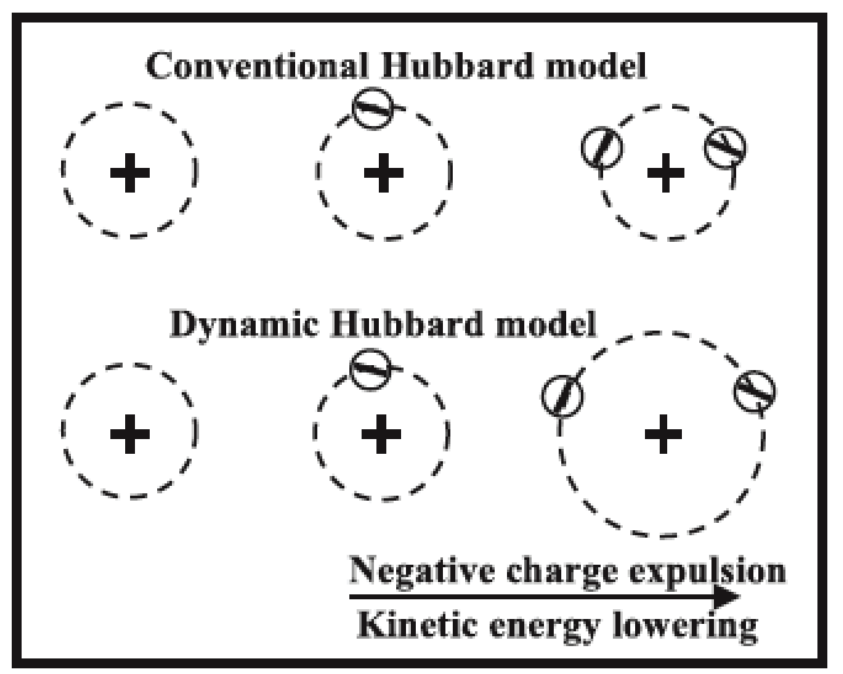

FIG. 3: Schematics of the physics described by dynamic Hubbard models

Various versions of dynamic Hubbard models can be constructed to embody this physics, involving auxiliary spin or local vibrational degrees of freedom, or in a purely electronic version a tight binding model with two rather than one orbital per site [25], as shown in Figs. 4 and 5 . The physics of all these models is very similar. Figure 6 illustrates the fact that holes have more difficulty propagating than electrons in these models. The low energy effective Hamiltonian that results from these models
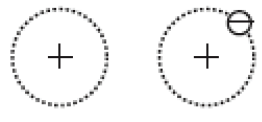

$|0\rangle \mid \rightarrow$

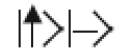

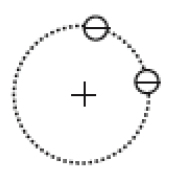

||$\downarrow|\downarrow|+|+\rangle$
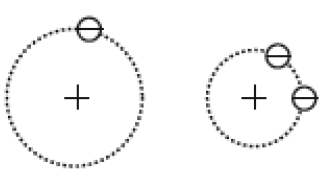

$|\uparrow\rangle|+\rangle$
FIG. 4: Site states of dynamic Hubbard model with an auxiliary spin degree of freedom with states $|+>|-,>$. The left three states (as well as $|\downarrow\rangle \mid->$ ) are lowest in energy and are the quasiparticle states in the low energy effective Hamiltonian with a correlated hopping term.

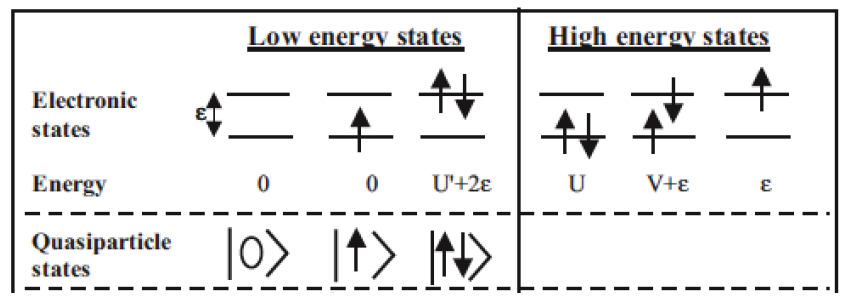

FIG. 5: Site states of dynamic Hubbard model with two orbitals per site. The low energy physics is identical to the one described by Fig. 4.

has a 'correlated hopping' term $\Delta t$ that gives different hopping amplitudes depending on the occupation of the sites involved in the hopping process and leads to kinetic energy driven pairing and superconductivity when the Fermi level is close to the top of the band, i.e. for hole carriers [26]. The $T_{c}$ versus hole concentration dependence gives the bell-shaped behavior characteristic of the cuprates as well as the $T_{c}$ versus e/a (electron/atom) ratio in transition metal alloys [27] (Matthias' rules) [28].
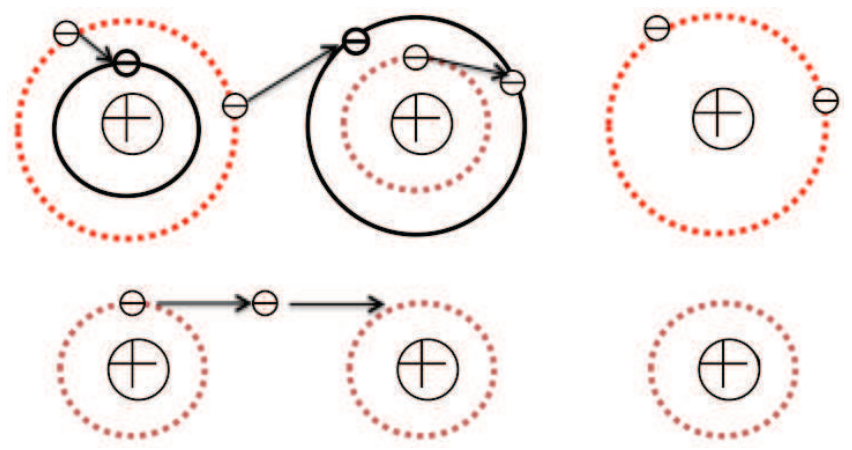

FIG. 6: Propagation of holes (upper pictures) versus propagation of electrons (lower pictures). Holes are highly dressed because they cause a large disruption in their environment as they propagate, electrons are undressed. In the upper picture the ful (dotted) circles denote the orbital in the final (initial) state.

The applicability of these models to high $T_{c}$ cuprates rests on the assumption that doped holes go into oxygen $p \pi$ orbitals in the plane [20], rather than $p \sigma$ orbitals as generally assumed. This assumption is supported by 
quantum chemical calculations by Goddard and coworkers [29]. We have recently argued that band structure calculations get this wrong because they do not take into account the energy lowering that results from local orbital relaxation when a hole goes into the doubly occupied $\mathrm{O}^{--} p \pi$ orbital [30]. These models also give rise to a strong tendency to charge inhomogeneity and phase separation due to the dominance of kinetic over potential energy [30], which correlates with behavior found in the cuprates [31].

\section{HOLE SUPERCONDUCTIVITY IN MATERIALS}

The models discussed in the previous section were introduced motivated by the physics of high $T_{c}$ cuprates. We found that they describe in a very natural way several salient properties of cuprate superconductors [12, 26, 32], in particular their:

(i) Dome-like $T_{c}$ versus hole concentration dependence

(ii) Positive pressure dependence of $T_{c}$

(iii) Crossover between strong and weak coupling regimes as the hole concentration increases

(iv) Crossover from incoherent to coherent behavior both as the hole concentration increases and as superconductivity sets in

(v) Tunneling asymmetry, with larger current for negatively biased sample

(vi) Apparent violation of conductivity sum rule, and transfer of optical spectral weight from high frequencies to low frequencies as superconductivity sets in.

In addition, we have argued [33] that these models lead to hole pairing and superconductivity in the following classes [34] of superconducting materials:

(1) Hole-doped cuprates [26, 32]

(2) Electron-doped cuprates [35]

(3) Magnesium diboride [36]

(4) Transition metal series alloys [27]

(5) Iron pnictides [37]

(6) Iron selenides [33]

(7) Doped semiconductors [33, 38]

(8) Elements under high pressure [39, 40]

(9) Sulphur hydride [41]

(10) A-15 materials [42, 43]

(11) All other superconductors [43]

For the simplest materials, the elements, there is an obvious preponderance of positive Hall coefficient for superconducting elements and negative Hall coefficients for nonsuperconducting elements [10, 44], as shown in Fig. 7. In the following we discuss the most fundamental reason that we believe makes holes indispensable for superconductivity.
Negative Hall coefficient=electron carriers

Positive Hall coefficient=hole carriers

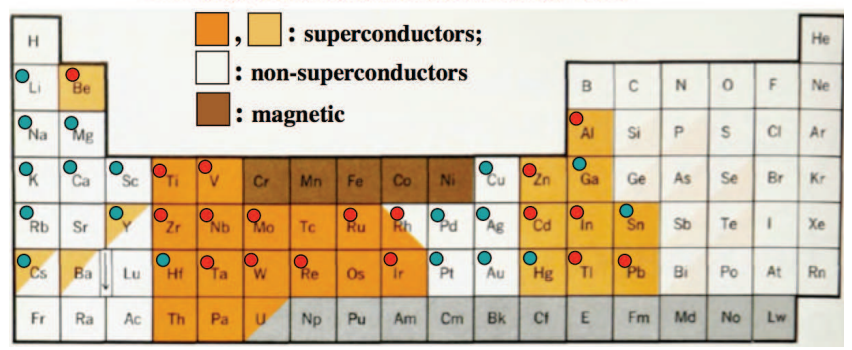

FIG. 7: Periodic table, showing the preponderance of superconductors among positive Hall coefficient elements and nonsuperconductors among negative Hall coefficient elements

\section{THE CENTRAL QUESTION IN SUPERCONDUCTIVITY}

I would like to propose that the central question in the phenomenon of superconductivity is a very basic one that even a child could ask, but scientists have never asked, nor answered. That question is the following:

When a superconductor in a magnetic field goes normal, how does the supercurrent stop?

I am assuming an ideal situation where the transition is perfectly reversible. For example, for given applied magnetic field $H<H_{c}$, the temperature is raised from slightly below $T_{c}(H)$ to slightly above $T_{c}(H)$. Alternatively, with the system at temperature $T_{c}(H)$, the magnetic field is raised from $H-\delta H$ to $H+\delta H$. When the system goes normal, the magnetic field penetrates the body and the supercurrent stops. Upon slightly cooling or slightly reducing the magnetic field, the supercurrent starts flowing again and the magnetic field is expelled.

How the supercurrent stops is a highly non-trivial question. In particular, what happens to its mechanical momentum [45]? The obvious and only possible answer is that the momentum of the supercurrent gets transferred to the body as a whole. For example, if the body is a cylinder hanging from a thread with a magnetic field along the direction of its axis, when the supercurrent stops the body will start to rotate around its axis.

This experiment has never been performed this way. However an equivalent experiment has been performed. If a magnetic field is applied to the superconductor, the current starts flowing in one direction and the body starts rotating in the same direction. The angular momentum of the body reflects the angular momentum of the supercurrent, carried by negative electrons moving in direction opposite to the current flow. In this situation, if the temperature is then raised, both the supercurrent and the rotation of the body will stop.

But how is the momentum of the supercurrent transferred to the body as a whole?

The problem is, the process is reversible. The reverse process is the Meissner effect. Any momentum transfer process involving collisions of electrons in the supercur- 
rent with phonons or impurities is an irreversible process, hence not allowed. We argue that the conventional theory of superconductivity cannot answer this question. The question has certainly never been posed nor answered in the superconductivity literature.

We have recently posed the question and proposed an answer to it [46]. The key element of the answer is holes. We argue that the only way that electrons can transfer mechanical momentum to the body as a whole in a reversible way is through the motion of holes.

\section{WHY HOLES ARE NOT LIKE ELECTRONS}

The velocity of Bloch electrons is given by

$$
\vec{v}_{k}=\frac{1}{\hbar} \frac{\partial \epsilon_{k}}{\partial \vec{k}}
$$

and the acceleration by

$$
\frac{d \vec{v}_{k}}{d t}=\frac{1}{\hbar^{2}} \frac{\partial^{2} \epsilon_{k}}{\partial \vec{k} \partial \vec{k}} \frac{\partial}{\partial t}(\hbar \vec{k})=\frac{1}{m_{k}^{*}} \frac{\partial}{\partial t}(\hbar \vec{k}) .
$$

The last equality is for the particular case of an isotropic band, with

$$
\frac{1}{m_{k}^{*}} \equiv \frac{1}{\hbar^{2}} \frac{\partial^{2} \epsilon_{k}}{\partial k^{2}}
$$

According to semiclassical transport theory, in the presence of an external force $\vec{F}_{\text {ext }}^{k}$

$$
\frac{\partial}{\partial t}(\hbar \vec{k})=\vec{F}_{e x t}^{k}
$$

The total force exerted on a Bloch electron is

$$
m_{e} \frac{d \vec{v}_{k}}{d t} \equiv \vec{F}_{\text {tot }}^{k}=\frac{m_{e}}{m_{k}^{*}} \vec{F}_{e x t}^{k}=\vec{F}_{e x t}^{k}+\vec{F}_{l a t t}^{k}
$$

with $m_{e}$ the bare electron mass, and $\vec{F}_{\text {latt }}^{k}$ the force exerted by the lattice on the electron of wavevector $k$, given by

$$
\vec{F}_{l a t t}^{k}=\left(\frac{m_{e}}{m_{k}^{*}}-1\right) \vec{F}_{e x t}^{k}
$$

Near the bottom of the band $m_{k}^{*}$ is positive and $\vec{F}_{\text {latt }}^{k}$ is small. Near the top of the band, $m_{k}^{*}$ is negative and $\vec{F}_{\text {latt }}^{k}$ is larger than $\vec{F}_{\text {ext }}^{k}$ and points in opposite direction, causing the electron near the top of the band to accelerate in direction opposite to the external force.

The importance of this for superconductivity is that when the lattice exerts a force on the electron, by Newton's third law the electron exerts a force on the lattice, or in other words transfers momentum to the lattice. This indicates that the electrons that are most effective in transfering momentum from the electrons to the body are electrons near the top of the band. In other words, holes. This will answer the central question of how the momentum of the supercurrent is transferred to the body as a whole in a reversible way, without energy dissipation.

\section{HOW HOLES ANSWER THE CENTRAL QUESTION IN SUPERCONDUCTIVITY}

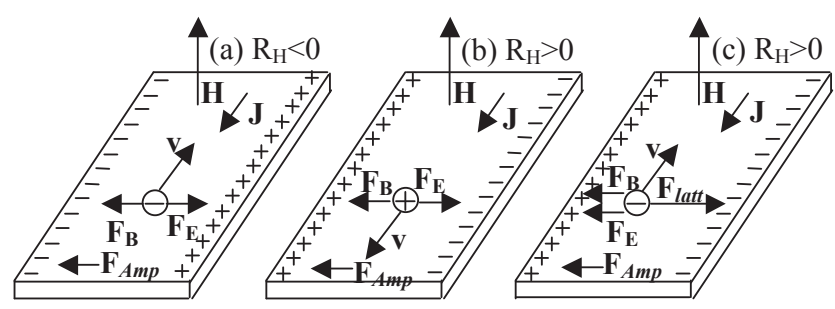

FIG. 8: Hall effect for a material with negative Hall coefficient (a) and for a material with positive Hall coefficient (b), (c). $F_{B}$ and $F_{E}$ are the magnetic and electric Lorentz forces acting on carriers, $F_{\text {latt }}$ is the force exerted by the lattice on the electron, $\vec{J}$ is the current density, $\vec{F}_{A m p}$ is the Amperian force on the bar.

Figure 8 shows the balance of forces on carriers in Hall bars with negative and positive Hall coefficients. For $R_{H}<0$, (Fig. $8(\mathrm{a})$ ), electric and magnetic forces on electrons are balanced, and for $R_{H}>0$, (Fig. 8 (b)), electric and magnetic forces on holes are balanced. However, in that case, electric and magnetic forces on electrons are not balanced, as shown in Fig. 8 (c)). For electrons to propagate along the direction of the current $\vec{J}$, another force is needed to balance electric and magnetic forces when $R_{H}>0$. That is the force exerted by the lattice on electrons, $F_{\text {latt }}$.

In the following, the forces under discussion are understood to be in direction perpendicular to the flow of the current $\vec{J}$ in Fig. 8. (There is also an electric force in direction parallel to $\vec{J}$ that is of no interest for the issue at hand). It is easy to see that the total force exerted by the lattice on the carriers is zero for a band close to empty with $R_{H}<0$ and is not zero for a band close to full and $R_{H}>0$ : the total force exerted by both the lattice and the external fields on the current carrying carriers has to be zero, hence from Eq. (5)

$$
\sum_{o c c} \vec{F}_{t o t}^{k}=\sum_{o c c} \frac{m_{e}}{m_{k}^{*}} \vec{F}_{e x t}^{k}=0
$$

where the sum is over occupied $k$ states. For the case $R_{H}<0$ and the band close to empty we can assume that the effective mass is independent of $k, m_{k}^{*}=m^{*}$. From Eq. (7)

$$
\sum_{o c c} \frac{m_{e}}{m_{k}^{*}} \vec{F}_{e x t}^{k}=\frac{m_{e}}{m^{*}} \sum_{o c c} \vec{F}_{e x t}^{k}=0
$$

therefore

$$
\sum_{o c c} \vec{F}_{e x t}^{k}=0
$$

and Eqs. (6), (8) and (9) imply

$$
\sum_{o c c} \vec{F}_{l a t t}^{k}=0
$$


so that the total force exerted by the lattice on the carriers in direction perpendicular to the current flow is zero, and so is the total force exerted by the carriers on the lattice.

Instead, for a band that is close to full and $R_{H}>0$, we cannot assume that $m_{k}^{*}$ is independent of $k$ for the occupied states, instead we assume $m_{k}^{*}=-m^{*}$ for the empty states. Eq. (7) then implies

$$
\sum_{o c c} \vec{F}_{t o t}^{k}=-\sum_{\text {unocc }} \frac{m_{e}}{m_{k}^{*}} \vec{F}_{e x t}^{k}=-\frac{m_{e}}{m^{*}} \sum_{\text {unocc }} \vec{F}_{\text {ext }}^{k}=0
$$

and from Eqs. (6) and (11)

$$
\begin{aligned}
\sum_{o c c} \vec{F}_{l a t t}^{k} & =-\sum_{o c c} \vec{F}_{e x t}^{k} \\
& =-\sum_{\text {all }} \vec{F}_{\text {ext }}^{k}+\sum_{\text {unocc }} \vec{F}_{e x t}^{k}=-2 N e \vec{E} \neq 0
\end{aligned}
$$

where $N$ is the number of $k$-points in the Brillouin zone. To obtain Eq. (12) we used that the external force is

$$
\vec{F}_{e x t}^{k}=e \vec{E}+\frac{e}{c} \vec{v}_{k} \times \vec{B}
$$

with $\vec{E}, \vec{B}$ the electric and magnetic fields.

Eq. (12) shows that when $R_{H}>0$ the lattice exerts a force on the conducting carriers that is perpendicular to the current flow. Conversely, the conducting carriers exert a force on the lattice or, in other words, transfer momentum to the lattice in direction perpendicular to the current flow. This force on the lattice, plus the electrostatic force on the positive ions that points in opposite direction (to the right in Fig. 8 (c)) gives the Amperian force on the Hall bar, $\vec{F}_{A m p}$. In contrast, if the carriers are electrons with $R_{H}<0$, the same $\vec{F}_{A m p}$ results from the direct force of the electric field on the ions (to the left in fig. 8 (a)) and there is no net force exerted by electrons on the lattice nor by the lattice on electrons in direction perpendicular to the current flow, hence no momentum transfer from the carriers to the lattice.

This is, in essence, why hole carriers are indispensable for superconductivity [47]. Let us next discuss how this explains the process of momentum transfer from the supercurrent to the body when the supercurrent stops.

Figure 9 shows schematically how the supercurrent stops when a cylindrical superconductor in a magnetic field pointing out of the paper goes normal. The inward motion of the N/S phase boundary is accompanied by a radial flow and counterflow of charge. In the process of becoming normal, superconducting electrons flow inward and are stopped by the clockwise magnetic Lorentz force resulting from this radial motion. At the same time normal holes flow inward and exert a torque on the body in the counterclockwise direction. The force $\vec{F}_{A m p}$ shown in the figure pointing counterclockwise is the same as the Amperian force in figure 7 (c) pointing left. In that way, the counterclockwise angular momentum possessed by the supercurrent is transferred to the body as a whole

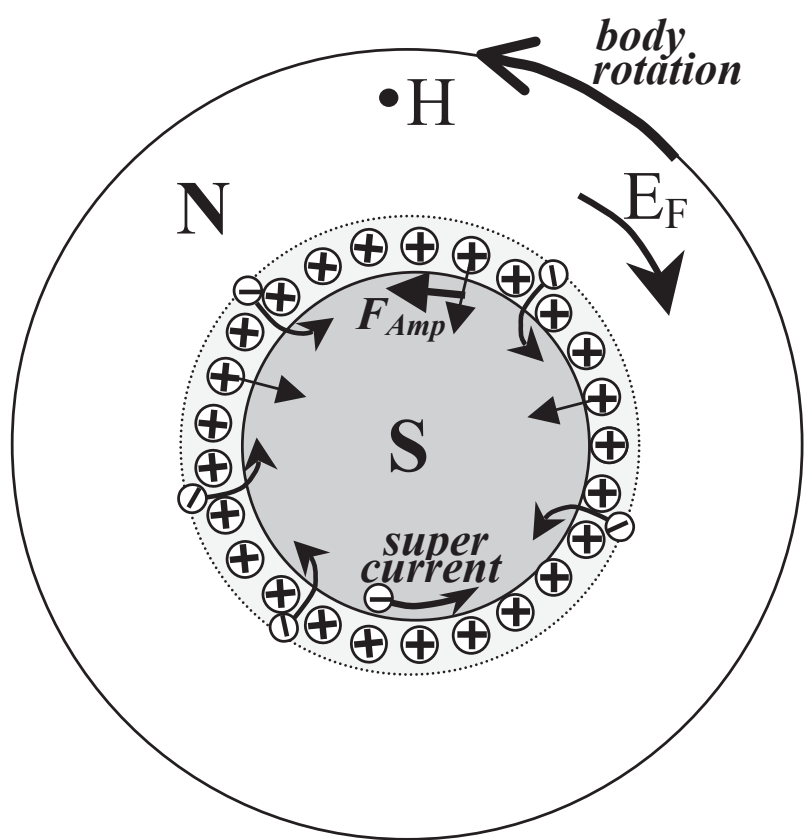

FIG. 9: Superconductor to normal transition in a magnetic field $H$ pointing out of the paper. Supercurrent flows clockwise, electrons carrying supercurrent move counterclockwise. The inward motion of the phase boundary is accompanied by inward flow of negative charge, that stops the supercurrent flow, and inward flow of normal hole carriers, that transfers the angular momentum of the supercurrent to the body as a whole that acquires counterclockwise rotation. Details are given in ref. [48].

without involving irreversible collisions that would dissipate Joule heat. In the reverse process where a normal cylinder becomes superconducting and expels the magnetic field, the direction of the motions in Fig. 9 are simply reversed (except for the direction of the supercurrent). The details of these processes are discussed in references $[47,48]$.

\section{DISCUSSION}

Alex Müller's and George Bednorz's breakthrough discovery in 1986 ushered in the era of hole superconductivity. Before their discovery, the term 'hole superconductor' had never been used, after their discovery it became commonplace. The evidence that hole carriers are necessary for superconductivity continues to accumulate. Of course sometimes it is the case that in a multiband situation electron carriers exist and dominate the transport, in which case it may not be obvious that hole carriers also exist and are responsible for superconductivity.

Possibly the one example where it is least obvious that hole carriers exist is for the very low carrier density ndoped semiconductor $\mathrm{SrTiO}_{3}$ [49-51]. It is believed that only electron carriers exist in this material, however ac- 
cording to the physics discussed in this paper superconductivity is impossible without hole carriers. Therefore, we conjecture that there is at least two-band conduction in this material [50] with one of the bands hole-like, the hole carriers would be induced by electron doping just like in the case of the electron-doped cuprates [35]. It is interesting that Alex Müller and coworkers in 1976 reported the finding of trapped holes near dopant impurities [52], which suggests that holes are easily induced in this material.

30 years after the discovery of the cuprate superconductors it is becoming increasingly clear that all superconductors are hole superconductors [53]. There are many reasons for this, all interconnected [12]. I believe the most fundamental reason is the one discussed in this paper, which can be summarized in the following very simple statements:

- In any superconductor, the mechanical momentum of the supercurrent has to be transferred to the body as a whole when the supercurrent stops.

- The process is reversible under ideal conditions.

- Only hole carriers can transfer mechanical momentum from electrons to the body as a whole in a reversible way, electron carriers cannot.

If this is so, it had been glimpsed at in the early days of superconductivity [10] but then was well hidden from superconductivity researchers for a long time, buried under the heavy weight of BCS theory, until Alex Müller's and George Bednorz's 1986 discovery of high $T_{c}$ superconductivity in cuprates started the process of bringing this deep secret of superconductors again into the open.

I am extremely grateful to them for having led me to this understanding. Happy 90th birthday Alex!
[1] K. A. Müller and J. Wieland, 'Apparatur zur Messung des Hall-Effektes und der magnetischen Widerstandsänderung mit Wechselstrom', Helv. Phys. Acta 27, 690 (1954).

[2] Furthermore, I just learned from Wikipedia that for his undergraduate diploma work, Müller worked on the Hall Effect in gray tin.

[3] J. G. Bednorz and K. A. Müller, Proc. 18th Int. Conf. on Low Temperature Physics, Kyoto, 1987, Jpn. J. Appl. Phys. 26, 1781 (1987) Supplement 26-3.

[4] K. A. Müller and J. G. Bednorz, Science 237, 1133 (1987).

[5] K. A. Müller, in "Mechanisms of High Temperature Superconductivity", Proceedings of the 2nd NEC Symposium, Hakone, Japan, October 2427, 1988, p. 2.

[6] H. Takagi, ref. [5], p. 238.

[7] Private communication, unfortunately I don't remember the source.

[8] Y. Tokura, H. Takagi, and S. Uchida, 'A superconducting copper oxide compound with electrons as the charge carriers', Nature (London) 377, 345 (1989).

[9] $\mathrm{Wu}$ Jiang et al 'Anomalous Transport Properties in Superconducting $\mathrm{Nd}_{1.85} \mathrm{Ce}_{0.15} \mathrm{CuO} \mathrm{O}_{4 \pm \delta}$, Phys. Rev. Lett. 73, 1291 (1994); P. Fournier et al, 'Thermomagnetic transport properties of $\mathrm{Nd}_{1.85} \mathrm{Ce}_{0.15} \mathrm{CuO}_{4+\delta}$ films: Evidence for two types of charge carriers', Phys.Rev. B 56, 14149 (1997); Y. Dagan and R.L. Greene, 'Hole superconductivity in the electron-doped superconductor $\mathrm{Pr}_{2-x} \mathrm{Ce}_{x} \mathrm{CuO} \mathrm{O}_{4}^{\prime}$, Phys. Rev. B76, 024506 (2007).

[10] K. Kikoin and B. Lasarew, 'Hall Effect and Superconductivity', Nature 129, 57 (1932); Physik. Zeits. d. Sowjetunion 3, 351 (1933); L. Brillouin, 'Le champ selfconsistent, pour des electrons lies; la supraconductibilite', Jour. de Phys. et le Rad. .VII, Tome IV, p. 333 (1933); A. Papapetrou, 'Bemerkungen zur Supraleitung', Z. f. Phys. 92, 513 (1934); M. Born and K.C. Cheng, 'Theory of Superconductivity', Nature 161, 968 (1948);
R. P. Feynman, 'Superfluidity and Superconductivity', Rev. Mod. Phys. 29, 205 (1957); I. M. Chapnik, 'On A Possible Criterion for Superconductivity', Sov. Phys. Dokl. 6, 988 (1962); 'On the empirical correlation between the superconducting $T_{c}$ and the Hall coefficient', Phys. Lett. A 72, (1979).

[11] J. E. Hirsch, 'Hole superconductivity', Phys. Lett. A 134, 4511989.

[12] References in http://physics.ucsd.edu/ jorge/hole.html.

[13] W. Heisenberg, 'Zum Paulischen Ausschlieungsprinzip', Annalen der Physik 402, 888 (1931).

[14] R. Peierls, 'Elektronentheorie der Metalle', Ergebnisse der exakten Naturwissenschaften Vol. 11, 284 (1932).

[15] R. Peierls. 'Zur Theorie der galvanomagnetischen Effekte', Z. für Physik 53, 255 (1929).

[16] J. E. Hirsch, 'Why holes are not like electrons. II. The role of the electron-ion interaction', Phys. Rev. B 71, 104522 (2005).

[17] J.G. Bednorz and K.A. Müller, 'Possible High $T_{c}$ Superconductivity in the $\mathrm{Ba}$ - La- Cu- 0 System', Z. Phys. B 64, 189 (1986).

[18] A. Bussmann-Holder, H. Keller and K. A. Müller, 'Evidences for polaron formation in cuprates', in Superconductivity in Complex Systems, p. 365, Springer, Berlin, 2005.

[19] H. Keller, , A. Bussmann-Holder and K. A. Müller, 'Jahn - Teller physics and high- $T_{c}$ superconductivity', Materials Today 11, 38 (2008).

[20] J. E. Hirsch and S. Tang, 'Hole superconductivity in oxides', Sol. St. Comm. 69, 987 (1989).

[21] J.E. Hirsch, 'Electron-hole asymmetric polarons', in "Polarons and Bipolarons in High- $T_{c}$ Superconductors and Related Materials", ed. by E. K. H. Salje, A. S. Alexandrov and W. Y. Liang, Cambridge University Press, Cambridge, 1995, p. 234; Physica C 201, 347 (1992).

[22] J. E. Hirsch, 'Polaronic superconductivity in the absence of electron-hole symmetry', Phys. Rev. B 47, 5351 (1993).

[23] J. E. Hirsch, 'Dynamic Hubbard Model', 
Phys. Rev. Lett. 87, 206402 (2001).

[24] J. E. Hirsch, 'Dynamic Hubbard model: kinetic energy driven charge expulsion, charge inhomogeneity, hole superconductivity and Meissner effect', Phys. Scr. 88, 035704 (2013).

[25] J. E. Hirsch, 'Why holes are not like electrons: A microscopic analysis of the differences between holes and electrons in condensed matter', Phys. Rev. B 65, 184502 (2002).

[26] J.E. Hirsch and F. Marsiglio, 'Superconducting state in an oxygen hole metal', Phys. Rev. B 39, 11515 (1989).

[27] J. E. Hirsch and F. Marsiglio, 'On the dependence of superconducting $T_{c}$ on carrier concentration', Phys. Lett. A 140, 122 (1989); X. Q. Hong and J. E. Hirsch, 'Superconductivity in the transition-metal series', Phys. Rev. B 46, 14702 (1992).

[28] B. T. Matthias, 'Transition Temperatures of Superconductors', Phys. Rev. 92, 874 (1953); 'Empirical Relation between Superconductivity and the Number of Valence Electrons per Atom', Phys. Rev. 97, 74 (1955).

[29] Y. Guo, J.M. Langlois and W.A. Goddard III, 'Electronic Structure and Valence-Bond Band Structure of Cuprate Superconducting Materials', Science 239, 896 (1988).

[30] J. E. Hirsch, 'Effect of orbital relaxation on the band structure of cuprate superconductors and implications for the superconductivity mechanism', Phys. Rev. B 90, 184515 (2014).

[31] A. Shengelaya and K. A. Müller, 'The intrinsic heterogeneity of superconductivity in the cuprates', EPL 109, 27001 (2015).

[32] F. Marsiglio and J.E. Hirsch, 'Hole superconductivity and the high- $T_{c}$ oxides', Phys. Rev. B 41, 6435 (1990).

[33] J.E. Hirsch, 'Materials and mechanisms of hole superconductivity', Physica C 472, 78 (2012) and references therein.

[34] 'Superconducting Materials: Conventional, Unconventional and Undetermined', Special Issue, Physica C 514 (2016); J.E. Hirsch, M.B. Maple, F. Marsiglio, 'Superconducting materials classes: Introduction and overview', Physica C 514, 1 (2016).

[35] J. E. Hirsch, 'Role of reduction process in the transport properties of electron-doped oxide superconductors', Physica C 243, 319 (1995).

[36] J.E. Hirsch, 'Hole superconductivity in $M g B_{2}$ : a high $T_{c}$ cuprate without Cu', Phys. Lett. A 282, 392 (2001); J.E. Hirsch and F. Marsiglio, 'Electronphonon or hole superconductivity in $M g B_{2}$ ?', Phys. Rev. B 64, 144523 (2001).

[37] F. Marsiglio and J.E. Hirsch, 'Hole superconductivity in arsenic - iron compounds', Physica C 468, 1047 (2008).

[38] E. Bustarret, 'Superconductivity in doped semiconductors', Physica C 514, 36 (2015).
[39] J.E. Hirsch and J.J. Hamlin, 'Why non-superconducting metallic elements become superconducting under high pressure', Physica C 470, S937 (2010).

[40] Jing Guo et al, 'The vital role of hole-carriers for superconductivity in pressurized black phosphorus', arXiv:1611.03330 (2016).

[41] J.E. Hirsch and F. Marsiglio, 'Hole superconductivity in $\mathrm{H}_{2} \mathrm{~S}$ and other sulfides under high pressure', Physica C 511, 45 (2015).

[42] A. A. Manuel et al, 'Contribution to the determination of the Fermi surface of $V_{3} S i$ by positron annihilation', Sol. St. Comm. 31, 955 (1979); S. Berko and M. Weger, 'Investigation of the Fermi Surface of $V_{3} S i$ by Means of Positron Annihilation', Phys. Rev. Lett. 24, 55 (1970); L. Hoffmann, A.K. Singh, H. Takei and N. Toyota, 'Fermi surfaces in $\mathrm{Nb}_{3} \mathrm{Sn}$ through positron annihilation', J. Phys. F 18, 2605 (1988).

[43] J.E. Hirsch, 'Bond-charge repulsion and hole superconductivity', $\quad$ Physica C 158, 326 (1989); 'Coulomb attraction between Bloch electrons', Phys, Lett. A 138, 83 (1989).

[44] J.E. Hirsch, 'Correlations between normalstate properties and superconductivity', Phys. Rev. B 55, 9007 (1997).

[45] J. E. Hirsch, "The missing angular momentum of superconductors", J. Phys. Cond. Matt. 20, 235233 (2008).

[46] J. E. Hirsch, 'On the reversibitity of the Meissner effect and the angular momentum puzzle', Annals of Physics 373, 230 (2016).

[47] J. E. Hirsch, 'Momentum of superconducting electrons and the explanation of the Meissner effect', Phys. Rev. B 95, 014503 (2017).

[48] J. E. Hirsch, 'The disappearing momentum of the supercurrent in the superconductor to normal phase transformation', Europhys. Lett. 114, 57001 (2016).

[49] J. F. Schooley et al, Phys. Rev. Lett. 14, 305 (1965).

[50] A. Bussmann-Holder , A. R. Bishop and A. Simon, ' $\mathrm{SrTiO}_{3}$ : From Quantum Paraelectric to Superconducting', Ferroelectrics 400, 19 (2010).

[51] X. Lin, Z.Zhu, B. Fauque, and K. Behnia, 'Fermi Surface of the Most Dilute Superconductor', Phys. Rev. X 3, 021002 (2013).

[52] O.F. Schirmer, W. Berlinger and K.A. Müller, 'Holes trapped near $\mathrm{Mg}^{2+}$ and $\mathrm{Al}^{3+}$ impurities in $\mathrm{SrTiO}_{3}$, Solid St. Comm. 18, 1505 (1976).

[53] J. E. Hirsch, 'Electron-hole asymmetry: the key to superconductivity', in "High Temperature Superconductivity: Physical Properties, Microscopic Theory, and Mechanisms", ed. by J. Ashkenazi et al, Springer, New York, 1991, p. 295. 